\title{
Intra and Inter Routing Based Lifetime Improvement Method for VFI Network-on-Chip
}

\author{
Ju Sung Kim, Jeong Beom Hong, and Tae Hee Han
}

\begin{abstract}
With the advent of the IoT age, low-power technology inside chips is a prerequisite. As lower power requirements have become important, network-on-chip (NoC) architecture has also begun to adopt design techniques that use a voltage frequency island (VFI), which is a low-power chip design paradigm. While research on maximizing the energy efficiency of NoC in the VFI environment has been performed, studies on the lifetime enhancement of the chip are scarce. In the previous research, the VFI routing scheme allocates a path that minimizes the VFI region change among the shortest paths because it considers only energy. However, if only the routing based on the minimum route is performed, a hotspot occurs owing to the traffic concentration, and the lifetime of the specific link is deteriorated rapidly. We herein propose a routing method that decreases the link failure probability and increases the lifetime in VFI NoC. Our algorithm uses different routing schemes for inter- and intra-communications to distribute traffic as much as possible. The proposed method is a routing method that can optimize energy consumption while focusing on traffic distribution using different routing methods according to each situation. This has resulted in an overall energy efficient load balancing and increased lifetime. The experimental results of mesh topology indicate that the proposed routing method increases the mean time to failure by $31.78 \%$ and the energy overhead is $4.45 \%$.
\end{abstract}

Index Terms-VFI NoC, lifetime improvement method, aging aware routing, LAXY routing.

\section{INTRODUCTION}

Moore's Law, which dominated the semiconductor industry for decades, is no longer applicable under the limitations of process development [1]. Instead of increasing the integration density of transistors, chip structures using multicore and manycore systems have been commercialized. To solve the problem with the increasing number of cores, a network-on-chip (NoC) architecture that exhibits advantages in scalability and parallelism has been spotlighted [2]. Voltage frequency island (VFI) is a low-power design technique to reduce the energy problems that arise from many cores and interconnections in the chip, and many studies have been conducted for its application to the NoC structure [3]-[6]. However, the high failure probability of the miniaturized transistor deteriorates the lifetime of the chip, and thus, this reliability problem has become important for the further development of the NoC structure.

Manuscript received January 9, 2019; revised March 15, 2019.

J. S. Kim is with Department of Electrical and Computer Engineering, Sungkyunkwan University, Suwon, Republic of Korea (e-mail: karlema@skku.edu).

J. B. Hong and T. H. Han are with Department of Semiconductor and Display Engineering, Sungkyunkwan University, Suwon, Republic of Korea, Department of Electrical and Computer Engineering, Sungkyunkwan University, Suwon, Republic of Korea (e-mail: \{adfffsa, than\}@skku.edu).
Nevertheless, studies on the reliability improvement of lowpower NoC considering VFI environment characteristics are scarce. In traditional VFI NoC research, many studies have been conducted on the regional segmentation method and the mapping method in NoC; however, studies on the routing-based scheme are scarce. In addition, only energyfocused studies have been conducted using the abovementioned methods, and research on reliability is scarce. In particular, the routing method in the VFI environment minimizes the number of passes through different VFI regions to reduce energy consumption during routing. However, applying energy-only routing increases the likelihood of failures owing to excessive traffic concentration on specific links. This accelerates the primary causes of chip failure such as negative bias temperature instability (NBTI) and time-dependent dielectric breakdown (TDDB) that reduce the mean time to failure (MTTF) [7], [8].

Therefore, in this study, we propose a technique to improve chip lifetime through load balancing. First, in VFI NoC, inter VFI communication between different VFI regions and intra VFI communication in the same region exist. Inter VFI communication differs from intra VFI communication because it contains synchronization logics at the boundary line. Therefore, our method divides inter and intra communications, and adopts different routing algorithms to distribute traffic energy efficiently. The LAXY routing technique is utilized in intra VFI communication, and a suitable configuration is set considering the size of the VFI region. If communication is performed between different VFI regions, we combine Dijkstra and adaptive routing to consider the lifetime and energy consumption. This approach has resulted in an overall energy efficient load balancing and increased lifetime.

\section{REALTED WORK}

\section{A. VFI Network-on-Chip}

A globally asynchronous, locally synchronous design technique is used for optimizing energy consumption. In the network-on-chip architecture, a VFI-based scheme has been introduced that divides the area within the chip and assigns different operating voltages and frequencies [9]. The design technique for applying VFI technology to network-on-chip consists of three steps. First, the partitioning process combines cores having similar operating voltages and frequencies. To decide the topology, workload information is extracted from task graphs between PEs and task scheduling is performed, and the initial values of the operating voltage and clock frequency are assigned to each 
core. Subsequently, considering the amount of communication and the energy consumption, we determine mathematically which cores are to be included in the same partition. Next, the topology of how tiles are laid out in the same VFI region is determined, and the routing techniques used in the NoC structure are also applied. In this process, the simple and well performing $\mathrm{XY}$ routing technique is utilized. Finally, when dividing a VFI NOC region, it receives the placement and routing information of the core and is assigned to the appropriate operating voltage and clock frequency of each VFI region. In conclusion, the results obtained in VFI NoC are shown in Fig. 1. By repeating the abovementioned series of processes, it leads to the optimal VFI NoC design. However, to allocate the voltage/frequency to the needs of each tile, a number of voltage and frequency synchronization logic, mixed clock FIFO (mcFIFO), and voltage level converter (VLC) are required. This synchronization logic results in increased chip energy consumption and design complexity. Therefore, to solve this problem, many techniques have been studied considering energy optimization when applying VFI technology to the NoC structure.

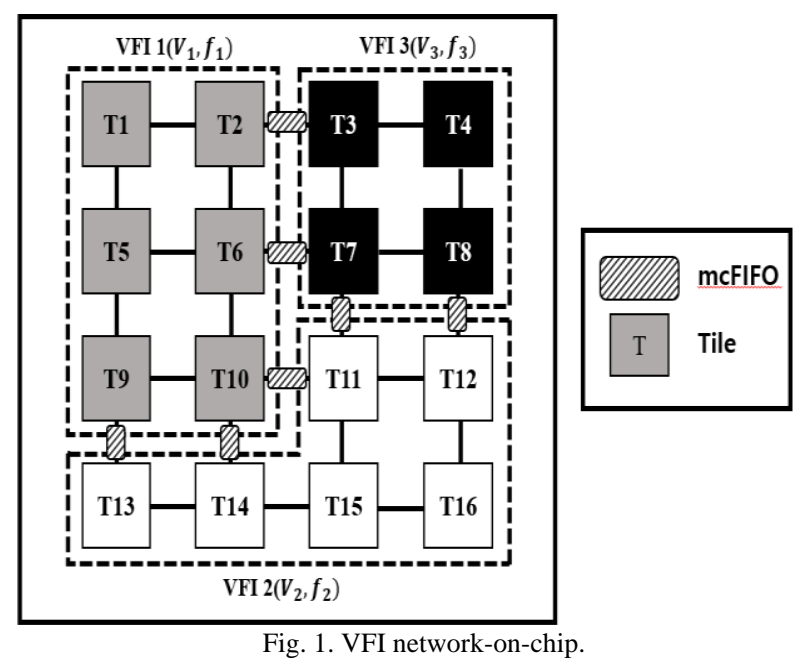

\section{B. LAXY Routing}

In previous research, two methods are used to reduce the fault caused by NBTI, HCI, and electromigration: a faulttolerant routing method detects a link or the router's fault and bypasses it [10]-[17] and adaptive aging avoidance (AAA) routing that distributes the amount of network traffic evenly [18]-[21]. The limitations of the AAA routing algorithm are twofold. First, an additional overhead of information transmission occurs because the traffic state must be known. Second, the design is considerably complicated. Because adaptive routing requires separate technologies for deadlock and live-lock handling, the router must be designed with these two techniques in mind. Recently, Rohbani [22] proposed a location-based agingresilient Xy-Yx (LAXY) routing algorithm to solve these drawbacks. The LAXY routing algorithm is simple in design and does not require additional traffic information when transmitting packets. In addition, the LAXY algorithm improves the disadvantages of the existing $X Y$ routing. As shown in Fig. 2, XY routing is a method to distribute the area statically because the traffic amount is concentrated in the NoC center area, and a hotspot zone is generated. It uses a specific configuration called Fishtail that distributes traffic using YX routing only in the west and east regions.

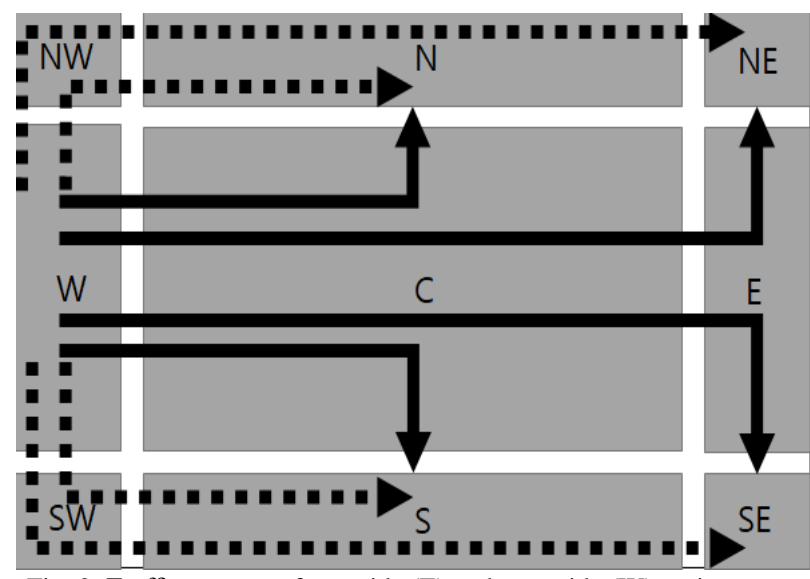

Fig. 2. Traffic amount of east side (E) and west side (W) regions over middle nodes $(\mathrm{C})$ of NoC using $\mathrm{XY}$ routing.

\section{LOAD BALNCING ROUTING ALGORITHM IN VFI NETWORK-ON-CHIP}

We herein propose a routing method considering the characteristics of the VFI environment applied to networkon-chip. Intra communications within the same VFI region utilize LAXY routing, a typical load balancing routing technique. However, considering the amount of communication between VFI regions, we utilize one of the various configurations that are used in the LAXY routing scheme. In inter VFI communication where different VFI regions must be passed through, it is typical to minimize the VFI region variations, considering the energy consumption and latency of the mcFIFO and VLC required for voltage and frequency synchronization. However, in our method, a mechanism that utilizes aging-aware adaptive routing based on the Dijkstra algorithm to prevent traffic from concentrating on a specific link is implemented. This mechanism is introduced in the next section.

\section{A. Inter VFI Communication Routing}

Inter VFI communication, unlike local Intra VFI communication within a VFI zone, requires relatively more hop counts as the routing proceeds on a chip-wide basis. Therefore, the inter VFI communication applies a larger amount of load to the entire chip, as compared to the Intra VFI communication that has a higher weight in determining the chip lifetime and energy. Therefore, we apply the inter routing scheme considering the chip lifetime first, and subsequently propose a technique to select the most suitable one among various configurations of LAXY routing in intra VFI communication according to the routing results. In general, if the VFI region must go through different voltages and frequency synchronization logics, it consumes additional energy and uses routing to reduce VFI region variations [3]. After obtaining all the paths with the minimum hop count as shown in Fig. 3 (a), the path with the minimum VFI region is selected. When routing from T15 to T9, as shown in Fig. 3 (c), the same hop count and mcFIFO are used. In previous research, to reduce hardware cost, several inter region routes share the same link as much as possible. However, this method can significantly reduce the 
lifetime of the chip because traffic is concentrated around specific links, such as the link between T10 and T11, as shown in Fig. 3(c). To distribute the traffic as much as possible, our method stores the amount of traffic on the links at the VFI region boundary and considers this value. When the hop count and number of mcFIFO are the same as shown in Fig. 3 (b), instead of sharing the already used inter-link, our scheme chooses a path considering the constraint condition determined by Eq. (4). We used the Dijkstra algorithm to obtain the shortest path. In this case, we implemented energy efficient load balancing routing by reflecting the energy cost as well as hop count to the cost value of the Dijkstra algorithm. The energy consumed in communication in NoC considering the VFI environment can be obtained as follows.

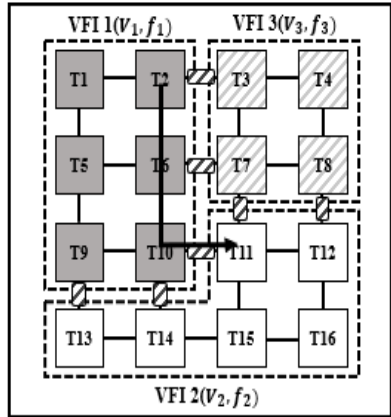

(a) VFI-aware Routing

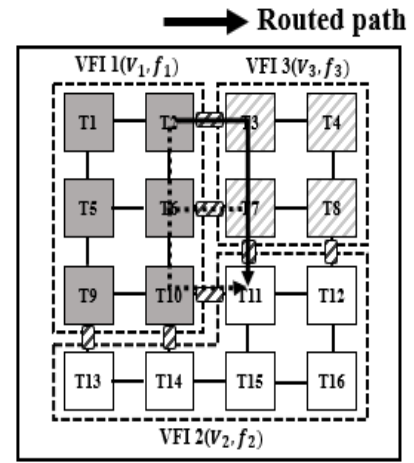

(b) Proposed Scheme

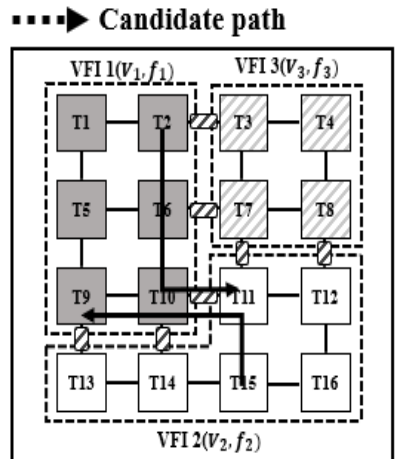

(c) When sharing an interlink using VFI-aware Routing

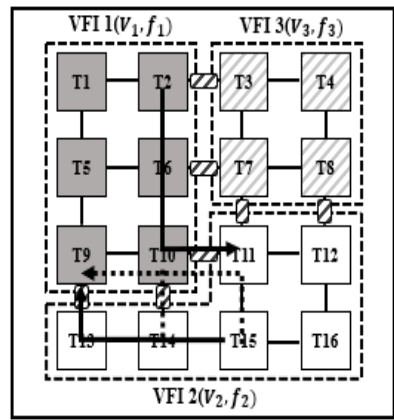

(d) Proposed Scheme

Fig. 3. Comparison between Inter VFI communication routing and proposed scheme.

$$
\mathrm{P}=\sum C_{\text {load }} V_{k}^{2} f_{k}
$$

$C_{\text {load }}$ represents the capacitance of the router and link in the communication process; $V_{k}^{2}$ and $f_{k}$ represent the operating voltage and clock frequency of the kth VFI region, respectively. Energy is affected only by the square of the operating voltage regardless of the clock frequency of each VFI region because the power is multiplied by time.

Therefore, the energy per bit consumed in VFI NoC communication is defined by Eq. (2).

$$
\begin{array}{r}
E_{b i t}\left(e_{i, j}\right)= \\
\sum_{p \in L\left(e_{i, j}\right)}\left(E_{L b i t}(p)+E_{B b i t}(p)+\right. \\
\left.E_{S b i t}(p)\right) \frac{V_{k}^{2}}{V_{D D}^{2}}
\end{array}
$$

and

$$
\begin{gathered}
\text { path }=\min _{\text {path }}\left[\alpha H+\sum_{\forall e_{i, j}}\left\{\operatorname{vol}\left(e_{i, j}\right) E_{b i t}\left(e_{i, j}\right)\right\}+\right. \\
\left.\sum_{q} E_{S Y N C}(q)\right]
\end{gathered}
$$

The shortest path is determined by Eq. (3). H represents the total hop count, and $\alpha$ is constantly proportional to energy. $E_{b i t}\left(e_{i, j}\right)$ denotes the energy consumed per bit of data transmitted from core i to core j. $E_{\text {Lbit }}(p), E_{B b i t}(p)$, and $E_{S b i t}(p)$ represent the energy consumed by the link, buffer, and switch measured at the reference voltage $V_{D D}^{2}$, respectively. The total bit energy is calculated according to the operating voltage of each VFI region. $E_{S Y N C}(q)$ is the energy consumed by the synchronization logic that includes the additional clock signal, VLC, and mcFIFO between different VFI regions.

After the path is determined by the Dijkstra algorithm, the links located at the boundary of the VFI region store the amount of traffic to be transmitted. If the same link is included in the routed path that is subsequently determined, it accumulates the amount of traffic. However, if the determined path does not satisfy the constraint of Eq. (4), another path must be considered.

$$
V_{l} \leq B W_{l}
$$

$B W_{l}$ represents the maximum bandwidth of link 1 on the routing path. If the link contained in the path determined by the Dijkstra algorithm violates the constraint, four routing path candidates are generated. If the start and end points are straight lines, the calculation is not performed and only the other cases are calculated. Select two routes in the four routing paths, depending on the cost, and select the route with the least number of routes through the link.

Further, if the cost is the same while violating the constraint, the path that does not share the inter-link path is selected. If all links violate the constraint, it sets the path with the smallest accumulated amount of traffic.

If all links in the path determined by the Dijkstra algorithm violate the constraint, the path with the smallest accumulated amount of traffic is chosen. Using this method, traffic is distributed, and the packet bottleneck is minimized.

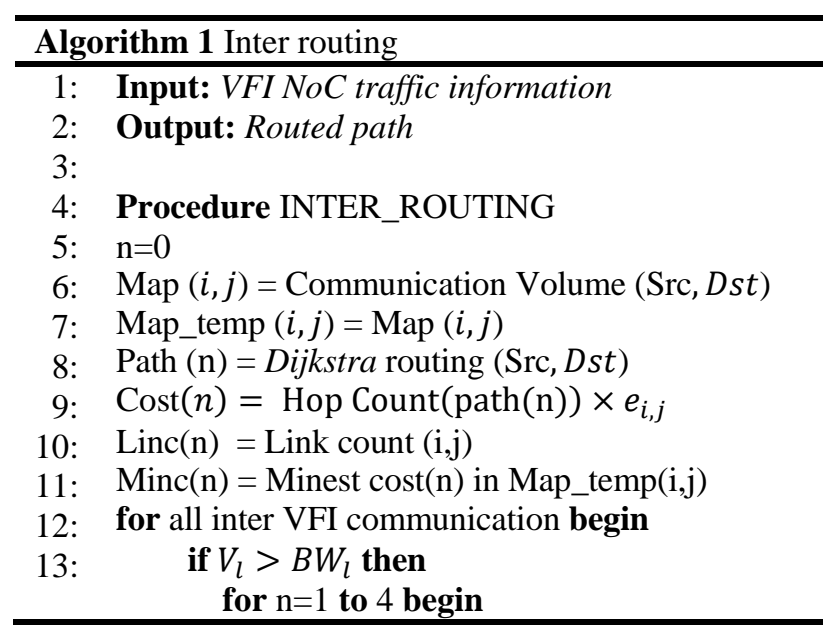




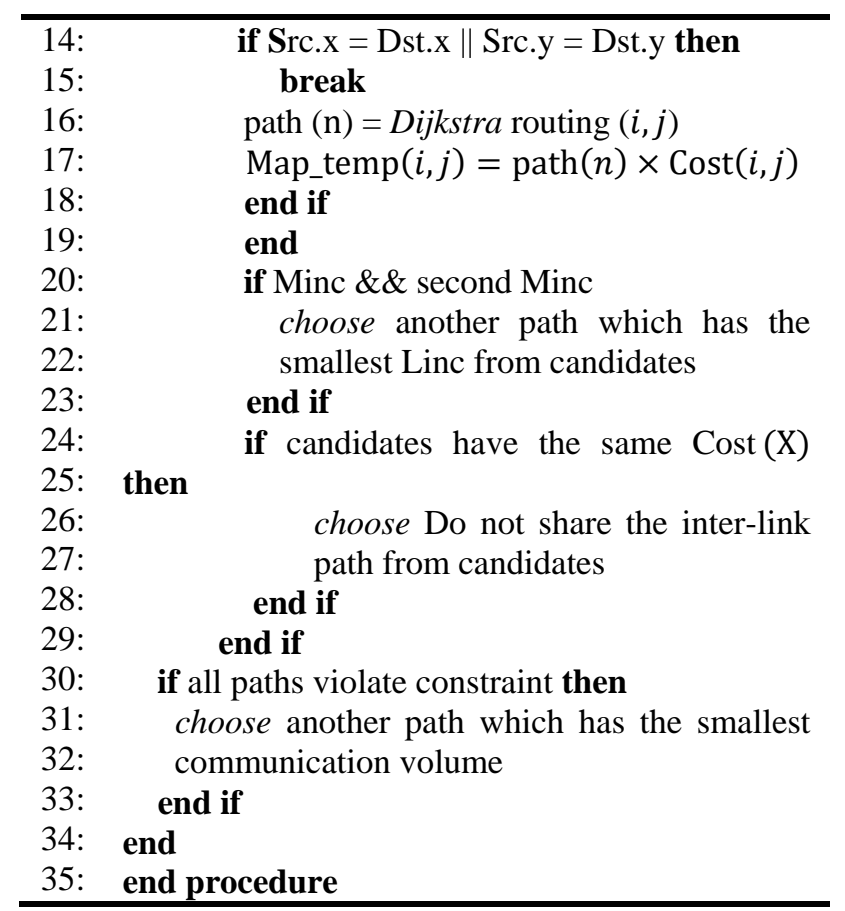

\section{B. Intra VFI Communication Routing}

Unlike inter-routing, intra-routing is the same as the general NoC structure without the VFI scheme because it does not go through mcFIFO, and it communicates within the region. In general, in the $\mathrm{NoC}$ architecture, $\mathrm{XY}$ routing is used primarily. $\mathrm{XY}$ routing is the least typical routing technique used in NoC designs, where packets are first routed along the $\mathrm{X}$ axis, arrive at the column at the destination tile, and subsequently reach the destination by routing to the $\mathrm{Y}$ axis. The advantage of $\mathrm{XY}$ routing is the NoC routing technique, which exhibits excellent performance with simple implementation complexity. However, although XY routing exhibits excellent performance, it is highly likely that traffic is concentrated on one side. To solve this problem, routing in the VFI region utilizes LAXY routing studied by Nezam Rohbani [22]. This LAXY routing divides the area within the chip and uses XY routing or YX routing depending on where the router is located. This LAXY routing has the effect of dispersing the traffic, compared to the basic XY routing that has no overhead, has and exhibits low latency and low complexity compared to aging-aware fully adaptive routing.

The LAXY technique uses different fishtail configurations depending on the size of the VFI region when dividing the area to which the YX routing is applied. The proposed method establishes different fishtail environments according to three conditions. Assuming an $\mathrm{N} \times \mathrm{M} \mathrm{VFI}$ region, if $\mathrm{N}$ and $\mathrm{M}$ are the same, an overall effective $\mathrm{N}-2, \mathrm{~N}$ $4, \mathrm{~N}-6, \mathrm{~N}-8$ fishtail configuration is used. Further, when $\mathrm{N}$ is larger than $\mathrm{M}$, we use the $\mathrm{N}-2, \mathrm{~N}-4,0,0$ configuration of LAXY because it expects the amount of communication to be higher as there are more routes in the $\mathrm{Y}$ direction than in the $\mathrm{X}$ direction in the inter-VFI communication region. If $\mathrm{M}$ is larger than $\mathrm{N}$, we use the $\mathrm{N}-4, \mathrm{~N}-6,0,0$ configuration of LAXY because it expects the amount of communication to be higher as there are more routes in the $\mathrm{X}$ direction than in the $\mathrm{Y}$ direction in the inter-VFI communication region. Hence, the inter-VFI communication is considered by predicting the amount of communication according to the width and height of the VFI region, and using different configurations of LAXY, thereby performing intra-routing according to the load balancing of the entire chip.

\begin{tabular}{|c|c|c|c|c|c|c|c|c|c|}
\hline 14400 & 35400 & 45600 & 48200 & 50400 & 54400 & 54400 & 47800 & 34800 & 15200 \\
\hline 22600 & 33800 & 45200 & 53600 & 59200 & 54800 & 55000 & 46600 & 37200 & 23400 \\
\hline 33000 & 46800 & 48800 & 50000 & 58000 & 58000 & 57600 & 51800 & 48600 & 37600 \\
\hline 39400 & 54200 & 48600 & 38600 & 48400 & 44600 & 47800 & 50000 & 52600 & 46200 \\
\hline 39800 & 61600 & 53200 & 42000 & 42200 & 40200 & 44200 & 49800 & 52600 & 50800 \\
\hline 41000 & 58800 & 56000 & 43800 & 44600 & 41600 & 40600 & 50600 & 53200 & 52600 \\
\hline 37800 & 52000 & 46600 & 48000 & 44200 & 42400 & 46600 & 52800 & 51200 & 51000 \\
\hline 32400 & 42400 & 37600 & 49600 & 53800 & 53600 & 54600 & 53800 & 43200 & 40600 \\
\hline 20600 & 43400 & 45600 & 61800 & 61600 & 62200 & 58400 & 48000 & 32400 & 25800 \\
\hline 13000 & 31800 & 38000 & 46600 & 51000 & 53400 & 52600 & 49400 & 29600 & 12600 \\
\hline
\end{tabular}

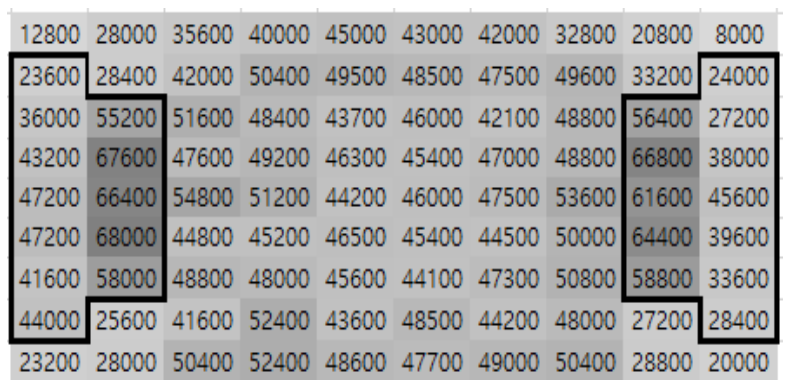

Fig. 5. When $N>M$, network traffic with $(8,6,0,0)$ configuration.

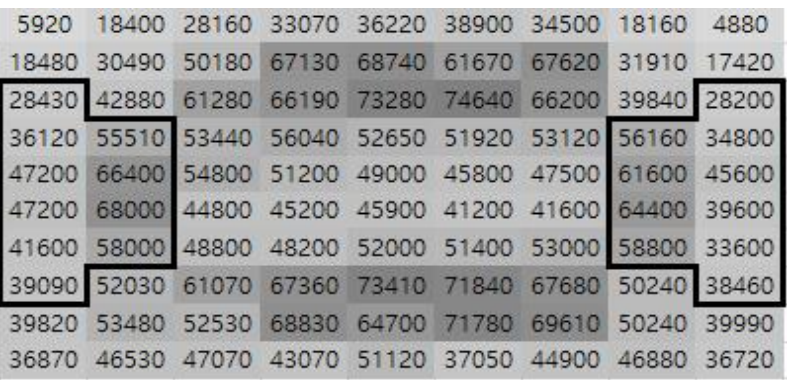

Fig. 6. When $\mathrm{N}<\mathrm{M}$, network traffic with $(6,4,0,0)$ configuration.

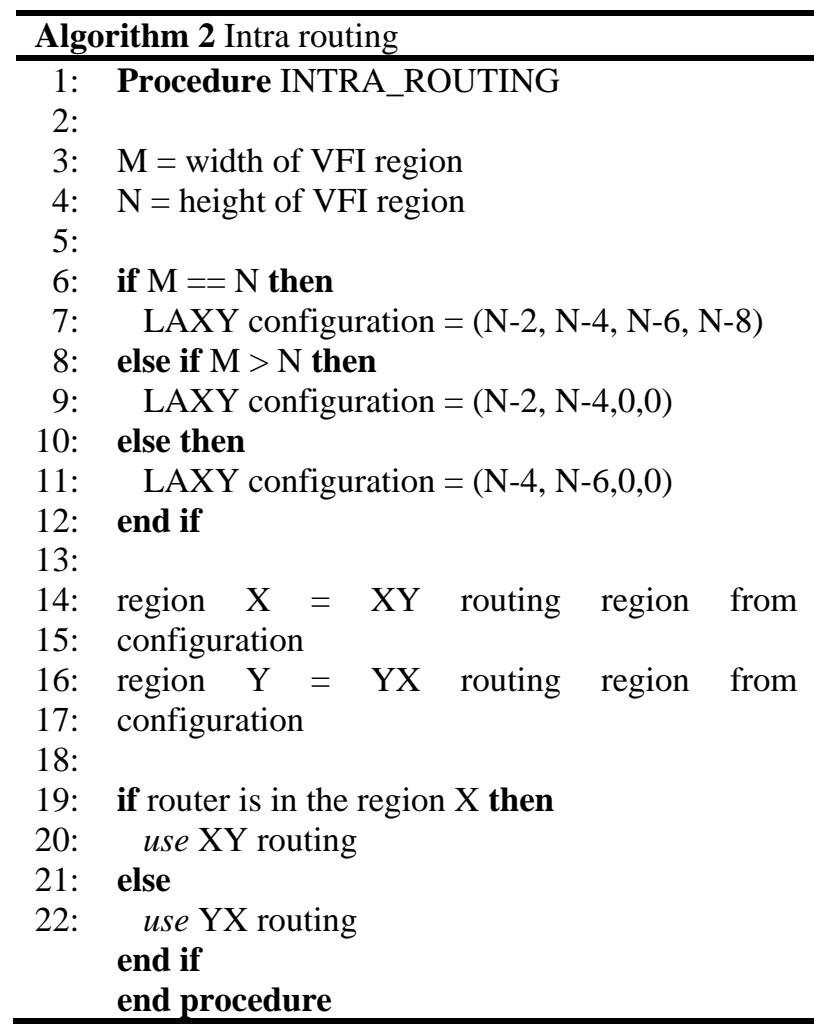




\section{LIFE TIME ESTIMATION FAILURE MODELS}

Owing to the increasing degree of integration in a chip, the influences of physical phenomena such as TDDB and NBTI that exhaust the lifetime of a transistor are increased. In NoC, the phenomena above become conspicuous on a link that carries a large amount of traffic. In particular, as the number of cores integrated in multicore and manycore technologies increases, the importance of enhancing the lifetime of chip also increases. TDDB is more influential owing to the reduction in the gate oxide thickness, and the breakdown of the oxide film is proportional to the temperature. NBTI can change the characteristics of a transistor when a high operating voltage or heat is applied to a specific area. If the communication volume is concentrated on a particular link, that link will be exposed to more heat than others. It increases the chip failure rate and reduces the overall lifetime and reliability of the chip. To predict the lifetime, mathematical modeling and simulation of the causes of chip lifetime degradation are performed in the microarchitecture and system design stage. The reliability aware microprocessor is a representative lifetime analytical method. It uses the MTTF model to estimate the lifetime. We analyze the improvement in lifetime by combining TDDB and NBTI effects with the sum-of-failure-rate model. The following equations are the MTTF of the TDDB and NBTI.

\section{A. Time Dependent Dielectric Breakdown (TDDB)}

TDDB is caused by a constant electric field. If the voltage is applied to the gate of the transistor, the tunneling current forms a conducting path in the oxide film. This path continues to expand over time and causes gate oxide breakdown eventually. Heat generation from excessive traffic in NoC causes TDDB much more rapidly. The MTTF model of TDDB is represented by Eq. (5).

$$
\mathrm{MTTF}_{T D D B} \propto\left(\frac{1}{V}\right)^{(a-b T)} e^{\frac{\left(x+\frac{Y}{T}+Z T\right)}{k T}}
$$

where the typically used values are as follows: $a=78, b=-$ $0.081, \mathrm{X}=0.759 \mathrm{eV}, \mathrm{Y}=-66.8 \mathrm{eVK}, \mathrm{Z}=-8.37 \mathrm{e}^{\wedge}(-4) \mathrm{eV} / \mathrm{K}, \mathrm{k}$ is Boltzmann's constant, and $\mathrm{T}$ is the operating temperature of the circuit based on the data from [7].

\section{B. Negative Bias Temperature Instability (NBTI)}

The NBTI is the physical phenomenon that changes the characteristics of the PMOS transistor. When a negative bias is applied to the gate of the PMOS, the threshold voltage increases and cannot be recovered fully. Thus, the transconductance and current of the transistor decrease gradually over time. Further, the high temperature accelerates the NBTI exponentially. The MTTF model of NBTI is expressed by Eq. (6).

$$
\operatorname{MTTF}_{N B T I} \propto\left[\ln \left(\frac{A}{1+2 e^{\frac{B}{k T}}}\right)-\ln \left(\frac{A}{1+2 e^{\frac{B}{k T}}-C}\right) \times \frac{T}{e^{\frac{D}{k T}}}\right]^{\frac{1}{\beta}}
$$

where $\mathrm{A}, \mathrm{B}, \mathrm{C}, \mathrm{D}$, and $\beta$ are model fitting parameters. Typical values of the parameters are as follows: $A=$ 1.6328, $B=0.07377, C=0.01, D=-0.06852 \quad$ and $\beta=0.3$ based on the data from [7], [8].

\section{ANALYSIS PERFORMANCE AND EXPERIMENTAL RESULTS}

\section{A. Energy Overhead Analysis in VFI NoC}

In VFI NoC, the total amount of energy consumed in communication is shown in Eq. (7), which is the sum of inter-communication energy and intra-communication energy [4].

$$
\begin{gathered}
E_{\text {total }}=E_{\text {inter }}+E_{\text {intra }} \\
E_{\text {inter }}=\sum_{k=1}^{T}\left(E_{\text {mcFIFo }} \times n_{k}^{\text {inter }}+E_{L} \times \sum_{i=1}^{M} n_{i, k}^{\text {intra }}\right. \\
\left.+E_{R} \times \sum_{i=1}^{M}\left(n_{i, k}^{\text {intra }}-1\right)\right)
\end{gathered}
$$

and

$$
E_{\text {intra }}=\sum_{k=1}^{Z}\left(E_{L} \times \sum_{i=1}^{M} n_{i, k}^{\text {intra }}+E_{R} \times \sum_{i=1}^{M}\left(n_{i, k}^{\text {intra }}-1\right)\right)
$$

In Eq. (8), $E_{\text {mcFIFO }}$ represents the energy consumption by mcFIFO inserted between different VFI regions, and it assumes that mcFIFO consumes $20 \%$ of the energy consumed by the connected router [4]. $E_{L}$ and $E_{R}$ represent the energy consumed by the link and the router, respectively. $n_{k}^{\text {inter }}$ represents the number of inter-links to be passed in inter-VFI communication, and in Eq. (9), $n_{i, k}^{\text {intra }}$ represents the number of links that pass through intra-VFI communication. $\mathrm{T}$ and $\mathrm{Z}$ are the numbers of inter-VFI communication and intra-VFI communication, respectively.

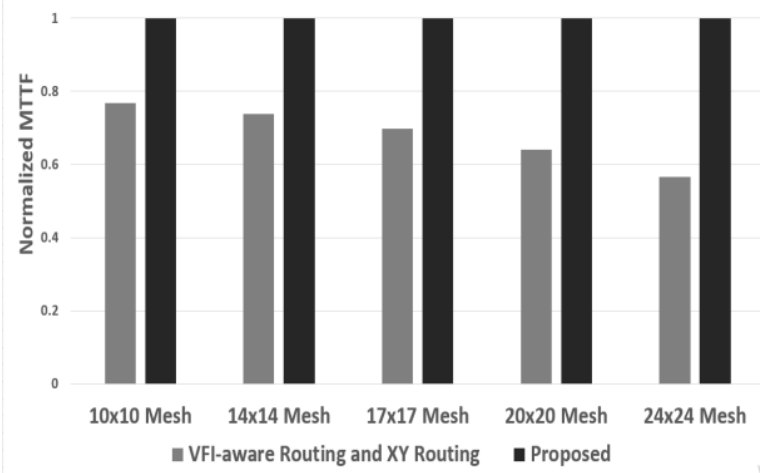

Fig. 7. The inter VFI-aware routing and INTRA XY routing are compared with proposed scheme MTTF results.

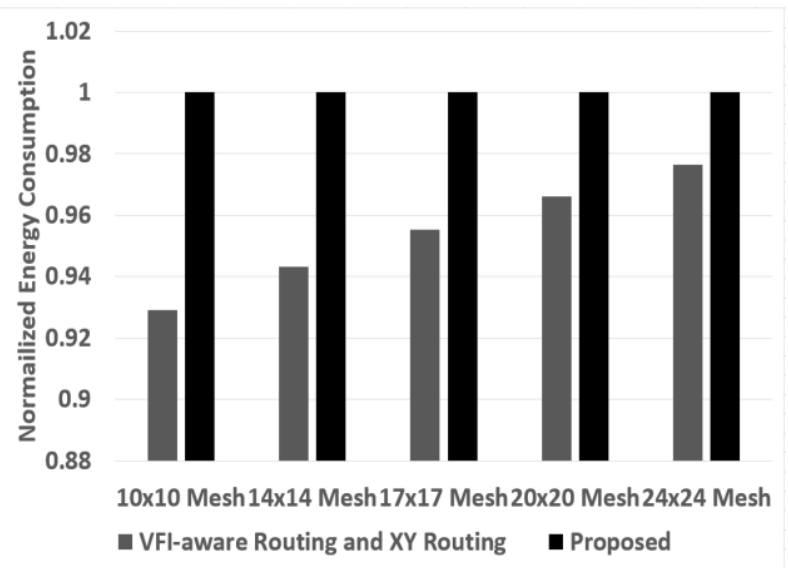

Fig. 8. The inter VFI-aware routing and intra XY routing are compared with proposed scheme energy consumption. 


\section{A. Experiment Results}

To measure the overall traffic volume and energy consumption in the optimal path and bypass path considering VFI NoC characteristics, NoXIM was used to verify the algorithm, calculate the amount of traffic, and model the energy consumed using Orion 2.0. To calculate the MTTF, the traffic temperature of $\mathrm{NoC}$ in the Hotspot 5.0 simulator was shown using the amount of NoXIM traffic and the energy value through Orion. Experiments were performed on 100 random traffics, and the same VFI NoC environment was constructed for a fair performance evaluation of each routing algorithm. Subsequently, $10 \times 10,14 \times 14,17 \times 17,20 \times 20$, $24 \times 24$ mesh topologies were used. To apply the optimal voltage frequency to each VFI region, the voltage/frequency of each region was divided into $(0.6 \mathrm{~V}, 0.78 \mathrm{GHz}),(0.7 \mathrm{~V}$, $1.27 \mathrm{GHz}),(0.8 \mathrm{~V}, 1.81 \mathrm{GHz}),(0.9 \mathrm{~V}, 2.42 \mathrm{GHz})$. The MTTF of the proposed algorithm is shown in Fig. 7. As the number of cores increases, the lifetime of the proposed routing increases by $31.78 \%$ on average compared to the method that combines VFI-aware routing and XY routing. Further, as shown in Fig. 8, the energy overhead decreased as the size of the mesh topology increased. The experimental result of averaging 100 runs using the simulator indicates that the energy overhead was $4.45 \%$.

\section{CONCLUSION}

Owing to the refinement of transistors, high failure rates exhausted the chip lifetime, and reliability has become an important problem. We herein proposed intra- and interrouting methods to improve the chip lifetime. The proposed method used different routing schemes for inter- and intraVFI communications, separately. For inter-VFI communication, our routing method focused on traffic distribution using the Dijkstra algorithm and the bandwidth constraint. In addition, different fishtail configurations in the LAXY algorithm were applied to intra-VFI communication. Our method increased the lifetime with little energy overhead in VFI NoC. Additionally, a larger mesh size improved the performance of the proposed method in terms of lifetime and energy.

\section{ACKNOWLEDGMENT}

This work was supported in part by the Basic Science Research Program through the National Research Foundation of Korea by the Ministry of Education under Grant NRF-2018R1D1A1B07043585

\section{REFERENCES}

[1] G. Venkatesh, J. Sampson, N. Goulding, S. Gracia, V. Bryksin, J. L. Martinez, S. Swanson, and M. B. Taylor, "Conservation cores: Reducing the energy of mature computations," ASPLOS, vol. 10, pp. 205-218, 2010.

[2] L. Benini and G. De Micheli, "Network on chips: A new SoC paradigm," Computer, vol. 35, no. 1, pp. 70-78, 2002.

[3] W. Y. Jang, D. Ding, and D. Z. Pan, "A voltage-frequency island aware energy optimization framework for networks-on-chip," IEEE Journal on Emerging and Selected Topics in Circuits and Systems, vol. 1, no. 3, pp. 420-432, Sept. 2011.
[4] C. L. Li, J. H. Lee, J. S. Yang, and T. H. Han, "Communication-aware custom topology generation for VFI network-on-chip," IEICE Electronics Express, vol. 11, no. 18, pp. 1-8, Sept. 2014.

[5] U. Ogras, R. Marculescr, D. Marculescu, and E. Jung, "Design and management of voltage frequency island partitioned networks-onchip," IEEE Trans. Very Large Scale Integration System, vol. 17, no. 3, pp. 330-341, March 2009.

[6] S. Herbert, S. Garg, and D. Marculescu, "Exploiting process variability in voltage/ frequency control," IEEE Trans. Very Large Scale Integration Systems, vol. 20, no. 8, pp. 1392-1404, Aug. 2009.

[7] J. Srinivasan, S. V. Adve, P. Bose, and J. A. Rivers, "The case for lifetime reliability-aware microprocessors," International Symposium on Computer Architecture, vol. 32, issue 2, pp. 276-287, March, 2004.

[8] S. Zafar, B. Lee, J. Stathis, A. Callegar, and T. Ning, "A model for negative bias temperature instability (NBTI) in oxide and high $\mathrm{k}$ pFETs," Int. Symposium on VLSI Technology, pp. 208-209, 2004.

[9] U. Y. Ogras, et al. "Voltage-frequency island partitioning for GALSbased networks-on-chip," in Proc. the 44th Annual Design Automation Conference, ACM, 2007.

[10] D. DiTomaso, A. Kodi, and A. Louri, "QORE: A fault tolerant network-on-chip architecture with power-efficient quad-function channel (QFC) buffers," in Proc. IEEE 20th Int. Symp. High Perform. Comput. Archit. (HPCA), Orlando, FL, USA, 2014, pp. 320-331.

[11] S. Pasricha and Y. Zou, "NS-FTR: A fault tolerant routing scheme for networks on chip with permanent and runtime intermittent faults," in Proc. 16th Asia South Pac. Design Autom. Conf. (ASPDAC), Yokohama, Japan, 2011, pp. 443-448.

[12] A. Hosseini, T. Ragheb, and Y. Massoud, "A fault-aware dynamic routing algorithm for on-chip networks," in Proc. IEEE Int. Symp. Circuits Syst. (ISCAS), Seattle, WA, USA, 2008, pp. 2653-2656.

[13] A. Vitkovskiy, V. Soteriou, and C. Nicopoulos, "A highly robust distributed fault-tolerant routing algorithm for NoCs with localized rerouting," in Proc. Interconnect. Netw. Archit. On-Chip Multi-Chip Workshop, New York, NY, USA, 2012, pp. 29-32.

[14] Y. Wang, L. Zhang, Y. Han, H. Li, and X. Li, "Address remapping for static NUCA in NoC-based degradable chip-multiprocessors," in Proc. IEEE 16th Pac. Rim Int. Symp. Depend. Comput. (PRDC), Tokyo, Japan, 2010, pp. 70-76.

[15] X. Li, Y. Cao, L. Wang, and T. Cai, "Fault-tolerant routing algorithm for network-on-chip based on dynamic XY routing," Wuhan Univ. J. Nat. Sci., vol. 14, no. 4, pp. 343-348, 2009.

[16] Y. Li and H. Gu, "Fault tolerant routing algorithm based on the artificial potential field model in network-on-chip," Appl. Math Comput., vol. 217, no. 7, pp. 3226-3235, 2010.

[17] M. Ali, M. Welzl, S. Hessler, and S. Hellebrand, “An efficient fault tolerant mechanism to deal with permanent and transient failures in a network on chip," Int. J. High Perform. Syst. Archit., vol. 1, no. 2, pp. 113-123, 2007.

[18] D. M. Ancajas, K. Bhardwaj, K. Chakraborty, and S. Roy, "Wearout resilience in NoCs through an aging aware adaptive routing algorithm," IEEE Trans. Very Large Scale Integr. (VLSI) Syst., vol. 23, no. 2, pp. 369-373, Feb. 2015.

[19] D. M. Ancajas, K. Chakraborty, and S. Roy, "Proactive aging management in heterogeneous NoCs through a criticality-driven routing approach," in Proc. Conf. Design Autom. Test Europe, Grenoble, France, 2013, pp. 1032-1037.

[20] K. Bhardwaj, K. Chakraborty, and S. Roy, "An MILP-based agingaware routing algorithm for NoCs," in Proc. Conf. Design Autom. Test Europe, Dresden, Germany, 2012, pp. 326-331.

[21] K. Bhardwaj, K. Chakraborty, and S. Roy, "Towards graceful aging degradation in NoCs through an adaptive routing algorithm," in Proc. 49th ACM/EDAC/IEEE Design Autom. Conf. (DAC), San Francisco, CA, USA, 2012, pp. 382-391.

[22] Rohbani, Nezam, et al. "LAXY: A location-based aging-resilient XyYx routing algorithm for network on chip," IEEE Transactions on Computer-Aided Design of Integrated Circuits and Systems, vol. 36, no. 10, pp. 1725-1738, 2017.

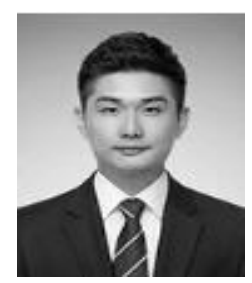

Ju Sung Kim received his BS degree in the information \& control engineering from Hankyung University, Ansung, Republic of Korea, in 2014, and now is a MS student in the Department of Electrical and Computer Engineering at Sungkyunkwan University, Suwon, Republic of Korea 


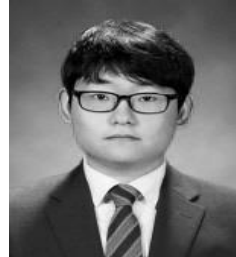

Jeong Beom Hong received his BS degree from the Department of Semiconductor and Display System Engineering from Sungkyunkwan University,

Suwon, Republic of Korea, in 2017, and now is a MS student in the Department of Semiconductor and Display Engineering at Sungkyunkwan University, Suwon, Republic of Korea

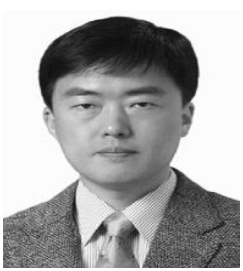

Tae Hee Han received his MS, and Ph.D. degrees in electrical engineering from KAIST, Daejeon, Republic of Korea, in 1992, 1994, and 1999, respectively. From 1999 to 2006, he had been with the Telecom R\&D Center in Samsung Electronics, Suwon, Korea. Since March 2008, he has been with Sungkyunkwan University, Suwon, Republic of Korea, as a professor. His research interests include SoC architectures and design technologies. From May 2011 to April 2013, he had served as a full-time advisor on semiconductor devices for the Korean government 